\title{
Tabiques no convencionales a base de residuos plásticos destinados a viviendas rurales
}

\author{
J.A. Zea ${ }^{1 *}$, D. Hernández ${ }^{1}$, J.E. Mandujano ${ }^{1}$, A. López ${ }^{1}$, P. Garnica ${ }^{2}$ \\ *Autor de Contacto: alex ze@ \\ ${ }^{1}$ Facultad de Ingeniería, Universidad Autónoma de Chiapas, México. \\ ${ }^{2}$ Centro de Investigación, Innovación y Desarrollo en Infraestructura y Seguridad Vial, México.
}

\section{RESUMEN}

En este trabajo se presenta el diseño y elaboración de un tabique no convencional adicionado con plástico tipo 1 PET, proveniente del reciclaje de las botellas plásticas de gaseosas como agregado principal dentro de la mezcla, que después de pasar por un proceso de fundición se plantea como un material aglutinante en la elaboración de tabiques. Por esto resulta importante estudiar sus características físicas y mecánicas, los resultados preliminares representan una alternativa viable para la fabricación de tabiques que se utilizan en el proceso de construcción de estructuras de mampostería destinados a viviendas rurales, donde se obtuvieron tabiques con resistencias superiores y comportamientos aceptables de acuerdo a lo requerido por las Normas vigentes.

Palabras clave: Tabique; Desechos; Plásticos; Mampostería; Viviendas.

\begin{abstract}
In this work, the design and elaboration of an unconventional partition added with type 1 PET plastic is presented, from the recycling of plastic soda bottles as the main aggregate within the mixture, which after going through a casting process is considered as a binder material in the manufacture of partitions. For this reason, it is important to study their physical and mechanical characteristics, the preliminary results represent a viable alternative for the manufacture of partitions that are used in the process of construction of masonry structures for rural dwelling, where partitions with superior resistance and acceptable behaviors were obtained in accordance with what is required by the current Standards.
\end{abstract}

Keywords: Brick; Waste; Plastics; Masonry; Dwelling.

\section{RESUMO}

Neste trabalho é apresentado o projeto e a elaboração de uma divisória não convencional acrescida de plástico PET tipo 1, a partir da reciclagem de garrafas plásticas de refrigerante como principal agregado da mistura, que após passar por um processo de fundição é considerada como um material ligante em a fabricação de partições. Por este motivo, é importante o estudo de suas características físicas e mecânicas, os resultados preliminares representam uma alternativa viável para a fabricação de divisórias que são utilizadas no processo de construção de estruturas de alvenaria para habitações rurais, onde partições com resistência superior e comportamentos aceitáveis foram obtidas de acordo com o que é exigido pelas Normas em vigor.

Palavras chave: Partição; Desperdício; Plásticos; Alvenaria; Habitações. 


\section{INTRODUCCIÓN}

Mundialmente aumenta el problema causado por la creciente cantidad de Residuos Sólidos Urbanos (RSU) y Residuos Sólidos Plásticos (RSP). En los últimos años a nivel mundial se ha generalizado el cuidado del medio ambiente y de recursos naturales; por esto, los materiales plásticos enfrentan hoy uno de los retos más importantes, ya que por sus ventajas de resistencia a la degradación y economía con respecto a otros elementos han sido cuestionadas por su impacto en el medio. (Morales, 2015)

A través del tiempo la industria del plástico ha ido en aumento y ha sustituido diversos materiales tales como el vidrio, la madera y el cloruro de polivinilo (PVC), por el tereftalato de polietileno (PET), ya que el mismo es un material caracterizado por su gran ligereza y resistencia mecánica a compresión, posee alto grado de transparencia y brillo, conserva el sabor y olor de los alimentos, $\mathrm{y}$ es una barrera contra los gases. El volumen total de todo el plástico producido es de 8.300 millones de toneladas, del cual $9 \%$ se recicla, $12 \%$ es incinerado y el $79 \%$ es acumulado en los vertederos o entornos naturales este último representa aproximadamente 6.300 millones de toneladas, esta gran cantidad de desechos fue impulsada por la vida moderna, donde el plástico se usa para muchos desechables o de "uso único", desde botellas de bebidas y pañales, hasta cubertería y cotonetes. (Alesmar et al., 2017)

Las botellas de bebidas son uno de los tipos más comunes de desechos plásticos siendo este de polietileno de alta densidad PET. En el 2016 se vendieron aproximadamente 4.80 billones de botellas de plásticos en todo el mundo, es decir un millón de botellas por minuto. El principal fabricante de botellas es el gigante de las bebidas coca cola la cual en el 2016 fabricó 110.000 millones. (BBC Mundo, 2017). El PET es uno de los materiales más útiles en la vida cotidiana, pero uno de los que genera más contaminación en el mundo. (Alesmar et al., 2017)

Es evidente la problemática actual de disposición de residuos de las ciudades y el interés de solucionarla, con el fin de evitar incrementos en la contaminación y daños ecológicos irreversibles. Con el fin de encontrar soluciones a esta preocupación, la tendencia en diversas investigaciones recientes, han enfocado sus esfuerzos en desarrollar no sólo técnicas y procesos constructivos novedosos, sino productos e insumos de construcción no convencionales, buscando reducir costos finales de proyectos e iniciando la búsqueda de alternativas y modificaciones a mezclas de concreto y mortero que lo permitan. (Criado, 2017)

En el presente estudio se propone el diseño y elaboración de un tabique añadido con materiales no convencionales, en donde destaca el plástico tipo 1 PET, proveniente del reciclaje de las botellas plásticas de gaseosas como un agregado aglutinante obtenido por un proceso de fundición del mismo, para utilizarse en el proceso de construcción de estructuras de mampostería destinados a viviendas rurales.

\section{PROCEDIMIENTO}

Los diseños de mezcla de los especímenes realizados se basaron en la determinación de la dosificación de cada uno de los materiales utilizados a través de ensayos "prueba-error" hasta lograr una mezcla óptima, que pudiera servir como material aglutinante en el caso específico y como un nuevo método de disposición final de los residuos de PET. Este estudio se enfocará en las pruebas físicas y mecánicas de un tabique no convencional adicionado con plástico tipo 1 PET conforme a los requisitos que estipulan las Normas Técnicas Complementarias para Diseño y Construcción de 
Estructuras de Mampostería (NTCM, 2017). Los parámetros a considerar en su diseño estarán basadas en características y propiedades de los tabiques de agregados convencionales o de cemento-arena estandarizados en el mercado.

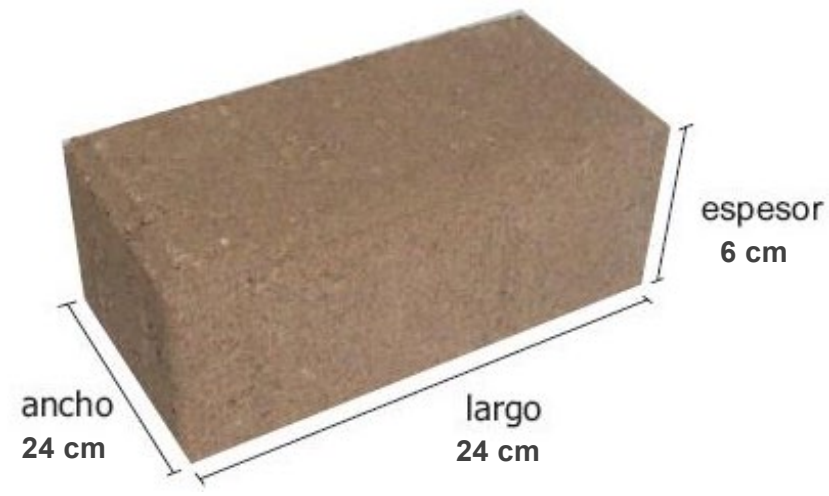

Figura 1.- Forma del tabique no convencional propuesto.

Fuente: Elaboración propia.

El tabique diseñado y elaborado cuenta con un volumen aproximado de $1728 \mathrm{~cm}^{3}$ (esto debido a que por proceso constructivo algunas dimensiones pueden variar).

\subsection{Proporcionamientos requeridos}

\subsubsection{Diseño experimental}

Se procedieron a elaborar tabiques no convencionales para lo cual se requirió contar con los siguientes materiales: plásticos reciclados (PET tipo 1), arena (tamiz $\mathrm{N}^{\circ} 4$ ), moldes para tabiques de $24 \times 12 \times 6 \mathrm{~cm}$, recipientes metálicos, horno de barro tradicional y materiales y equipo necesarios para su funcionamiento y equipo básico del Laboratorio de Tecnología del Concreto para ensayos a compresión (axial, axial en pilas, diagonal y absorción).

Se recolectaron botellas plásticas de PET, de bebidas gaseosas, en la Facultad de Ingeniería de la Universidad Autónoma de Chiapas. Un aproximado de 170 kilogramos (5700 botellas), de las cuáles se estima un $10 \%$ de desperdicio aproximado durante el proceso de fundición. Las botellas fueron limpiadas y preparadas para seguir el procedimiento que se muestra en la Figura 2. Las botellas de PET fueron derretidas en un recipiente metálico, el cual se introducía en un horno de barro convencional, tradicional. La temperatura a la cual se fusionaba el PET oscilaba entre $250 \mathrm{y}$ $300{ }^{\circ} \mathrm{C}$. En el caso del agregado fíno se empleó arena, proveniente del Río Santo Domingo de la Ciudad de Chiapa de Corzo, Chiapas, México. La arena fue tamizada para pasar la malla No. 4 (4.76 mm, tamaño de partícula). Se fabricaron moldes de acero, con una dimensión de 24x12x6 $\mathrm{cm}$.

Debido a que en la literatura no se encuentra mucha información del uso del PET tipo 1, en forma de aglutinante (derretido), en componentes constructivos, para la fabricación de los tabiques se propuso una cantidad de material inicial en base a proporciones de tipo empírica e intuitiva en la fabricación de éstos mismos, pero proponiendo como material aglutinante plástico tipo 1 PET. La cantidad de PET establecida varía en un 18-25\% dentro de la mezcla; por tal motivo, para un volumen de mezcla de $1728 \mathrm{~cm}^{3}$ que equivale al volumen de un tabique, fijando así una proporción óptima del 22\% de PET respecto del agregado fino. 
Además se procedió a implementar la metodología siguiente:
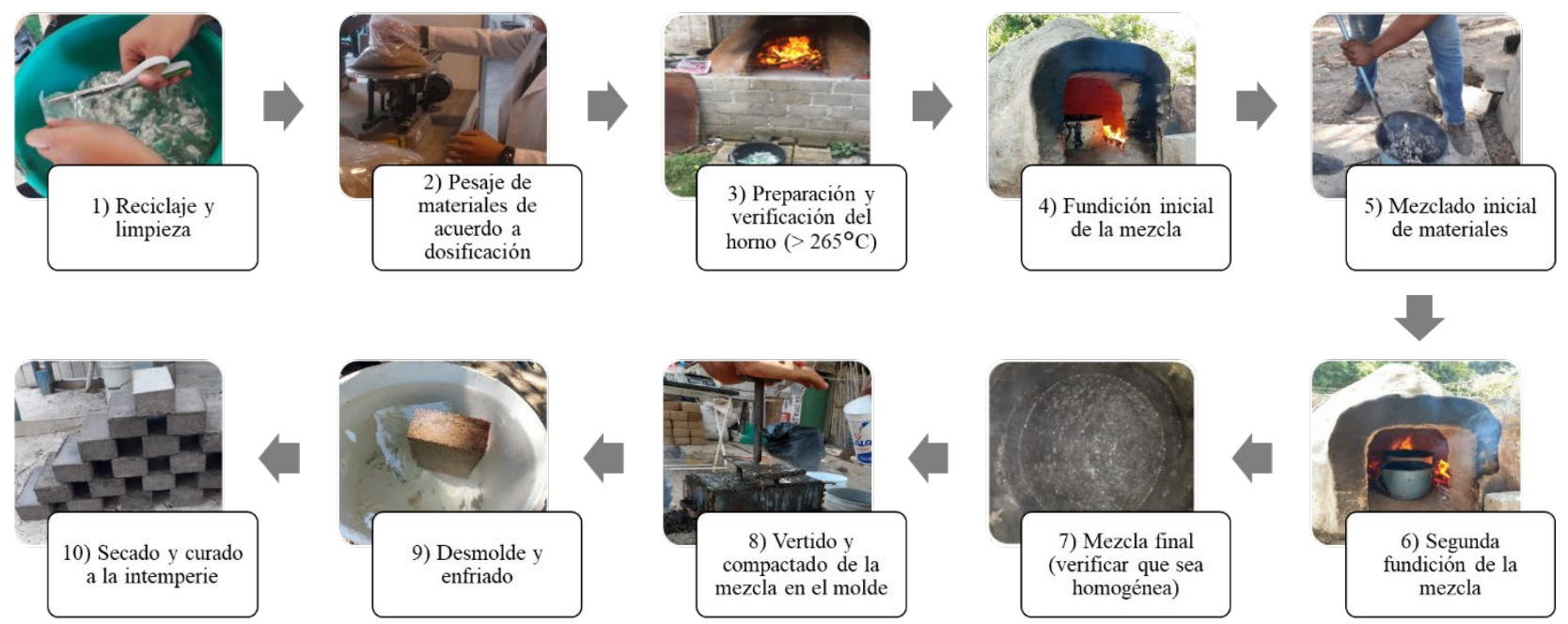

Figura 3.- Secuencia del proceso de fabricación de los tabiques no convencionales Fuente: Toma propia

\subsubsection{Preparación de especímenes}

Para este estudio se prepararon en total 155 tabiques (piezas) para las pruebas de resistencia a compresión axial, absorción y humedad, compresión axial en pilas y compresión diagonal (cortante) en muretes.

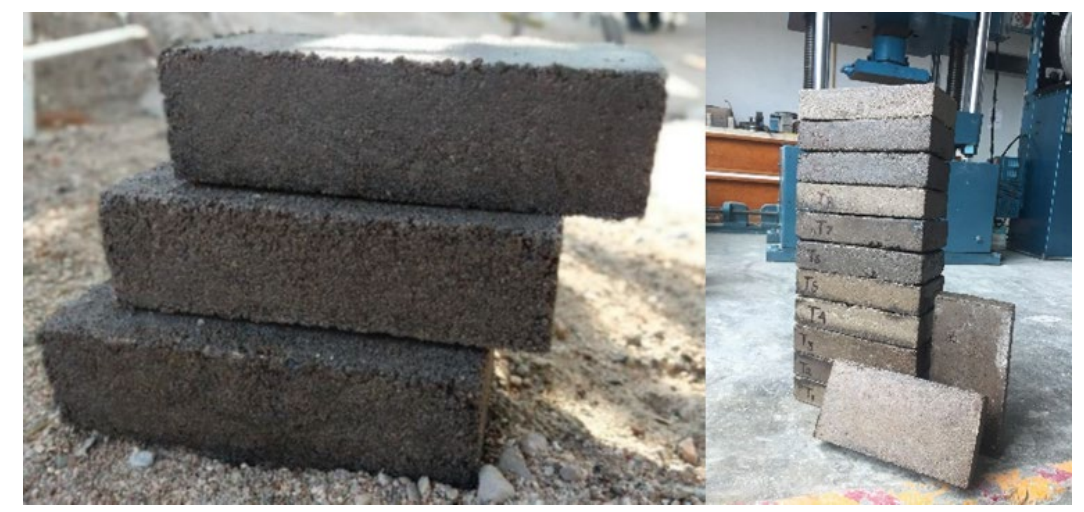

Figura 4.- Tabiques no convencionales

Fuente: Toma propia.

Tabla 1.- Características de los especímenes y tiempos de prueba para ensayos mecánicos

\begin{tabular}{|cccc|}
\hline Prueba & Descripción & $\begin{array}{c}\text { Edad de prueba } \\
\text { (días) }\end{array}$ & $\begin{array}{c}\text { Número de } \\
\text { especímenes }\end{array}$ \\
\hline $\begin{array}{c}\text { Resistencia a compresión } \\
\text { simple (piezas) }\end{array}$ & Tabiques: $240 \mathrm{~mm} \times 120 \mathrm{~mm} \times 60 \mathrm{~mm}$ & 28 & 28 \\
\hline $\begin{array}{c}\text { Resistencia a compresión axial } \\
\text { en pilas }\end{array}$ & Altura: $43 \mathrm{~cm}-44 \mathrm{~cm}$ & 28 & 10 \\
\hline $\begin{array}{c}\text { Resistencia a compresión } \\
\text { diagonal en muretes }\end{array}$ & Diagonal: $50.50 \mathrm{~cm}-51.00 \mathrm{~cm}$ & 28 & 10 \\
\hline
\end{tabular}

Fuente: Elaboración propia 
Los especímenes fueron ensayados en el Laboratorio de la Dirección General de Servicios Técnicos de la Secretaría de Comunicaciones y Transportes (SCT), Delegación Chiapas. Después de fabricar los especímenes se dejaron enfriar, secar y curar por $24 \mathrm{~h}$ a la intemperie. Las edades de ensayo y el número de piezas para cada prueba considerada se muestran en la Tabla 1.

\subsubsection{Caracterización de los especímenes en estado endurecido}

\section{Resistencia a la compresión}

Para los ensayos en especímenes individuales (piezas) se siguió lo establecido en la NMX-C-404ONNCCE-2012 (Industria de la construcción - Bloques, tabiques o ladrillo tabicones para uso estructural - Especificaciones y métodos de ensayo) y para el caso de pilas y muretes se siguió lo establecido a la NMX-C-464-ONNCCE-2010 (Industria de la construcción - Mampostería Determinación de la resistencia a compresión diagonal y módulo de cortante de muretes y la resistencia a compresión y módulo de elasticidad de pilas de mampostería de arcilla o de concreto - Métodos de ensayo). En total se emplearon 20 piezas individuales, 10 pilas y 10 muretes, tal y como se ilustra en la Figura 5.

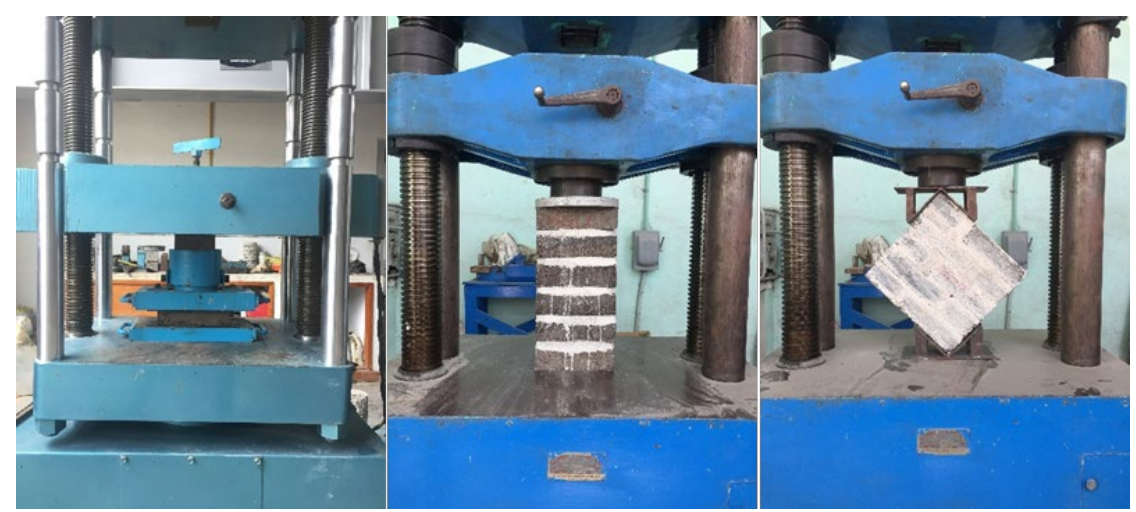

Figura 5.- Prueba de resistencia a compresión (Izquierda a derecha:

Compresión axial por piezas, compresión axial en pilas, compresión diagonal en muretes) Fuente: Toma propia.

\section{RESULTADOS}

\subsection{Características de los especímenes}

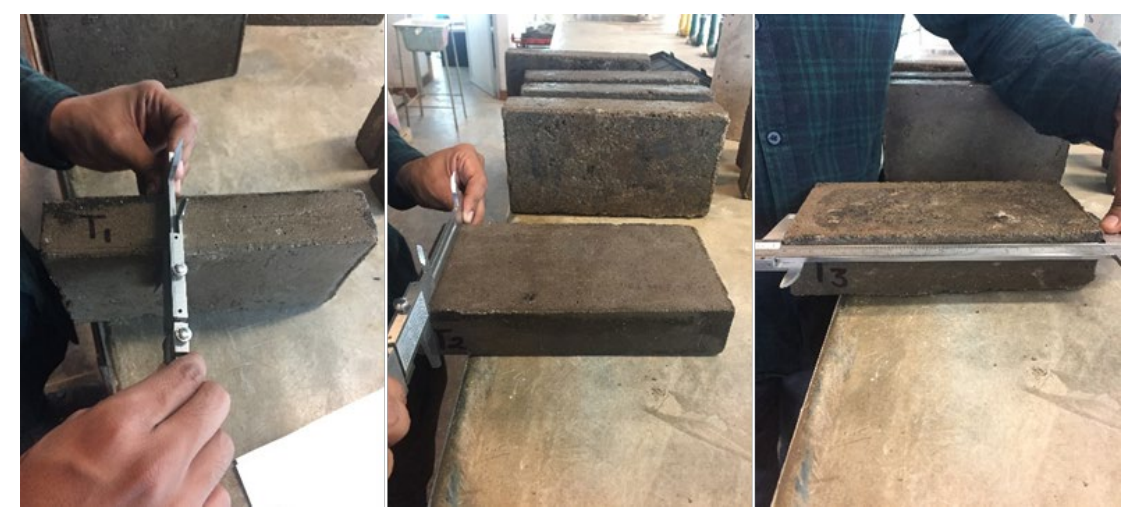

Figura 6.- Medición de las dimensiones de la pieza

Fuente: Toma propia 
Una vez, reposado los tabiques no convencionales por 28 días de secado a la intemperie, se procedieron a medir las dimensiones y peso de cada una de los especímenes. En la Tabla 2 se muestran cada una de las características geométricas de las piezas.

\subsection{Prueba de humedad y absorción}

Determinación de la absorción de agua y absorción inicial de agua NMX-C-037-ONNCCE. Para absorción total en 24 horas: Se registran las masas de los 5 especímenes ya secos y se sumergen en agua por un periodo de 24 horas. Terminado este periodo se sacan y se elimina el agua superficial con un paño o papel absorbente; se seca también el interior de las celdas, y se vuelve a determinar su masa. (Instituto Mexicano del cemento y concreto, A.C, 2014). Los datos son listados en la Tabla 2 y mostrados en la gráfica de la Figura 7.

Tabla 2.- Características de los especímenes

\begin{tabular}{|ccccccc|}
\hline No. de muestra & $\begin{array}{c}\text { Largo, } \\
\mathbf{c m}\end{array}$ & $\begin{array}{c}\text { Ancho, } \\
\mathbf{c m}\end{array}$ & $\begin{array}{c}\text { Espesor, } \\
\mathbf{c m}\end{array}$ & $\begin{array}{c}\text { Peso Seco, } \\
\mathbf{k g}\end{array}$ & $\begin{array}{c}\text { Peso Húmedo, } \\
\mathbf{k g}\end{array}$ & $\begin{array}{c}\text { Porcentaje de } \\
\text { absorción, } \\
\mathbf{\%}\end{array}$ \\
\hline 1 & 23.9 & 12.1 & 6.0 & 3.712 & 3.777 & 1.74 \\
\hline 2 & 23.9 & 11.9 & 5.9 & 3.810 & 3.898 & 2.30 \\
\hline 3 & 24.1 & 11.9 & 5.9 & 3.550 & 3.643 & 2.63 \\
\hline 4 & 23.9 & 11.8 & 6.0 & 3.677 & 3.766 & 2.41 \\
\hline 5 & 23.8 & 12.0 & 5.9 & 3.796 & 3.869 & 1.92 \\
\hline 6 & 24.0 & 11.9 & 6.0 & 3.652 & 3.725 & 2.01 \\
\hline 7 & 23.8 & 12.0 & 6.0 & 3.546 & 3.624 & 2.21 \\
\hline 8 & 23.8 & 12.0 & 5.9 & 3.686 & 3.771 & 2.31 \\
\hline 9 & 23.9 & 12.0 & 5.9 & 3.622 & 3.710 & 2.43 \\
\hline 10 & 24.1 & 11.9 & 6.0 & 3.756 & 3.852 & 2.56 \\
\hline 11 & 23.8 & 11.9 & 6.1 & 3.714 & 3.798 & 2.27 \\
\hline 12 & 23.9 & 11.8 & 5.9 & 3.822 & 3.901 & 2.08 \\
\hline 13 & 23.8 & 12.0 & 6.0 & 3.766 & 3.854 & 2.33 \\
\hline 14 & 23.8 & 11.9 & 6.0 & 3.586 & 3.656 & 1.94 \\
\hline 15 & 23.9 & 12.0 & 5.9 & 3.732 & 3.817 & 2.29 \\
\hline 16 & 24.1 & 11.9 & 6.0 & 3.816 & 3.913 & 2.53 \\
\hline 17 & 24.1 & 11.9 & 6.1 & 3.560 & 3.649 & 2.49 \\
\hline 18 & 23.9 & 11.9 & 5.9 & 3.814 & 3.898 & 2.19 \\
\hline 19 & 24.1 & 11.8 & 6.0 & 3.731 & 3.803 & 1.93 \\
\hline 20 & 23.8 & 12.0 & 6.0 & 3.792 & 3.882 & 2.37 \\
\hline
\end{tabular}

Fuente: Resultados obtenidos en el Laboratorio de la Facultad de Ingeniería, UNACH, Chiapas.

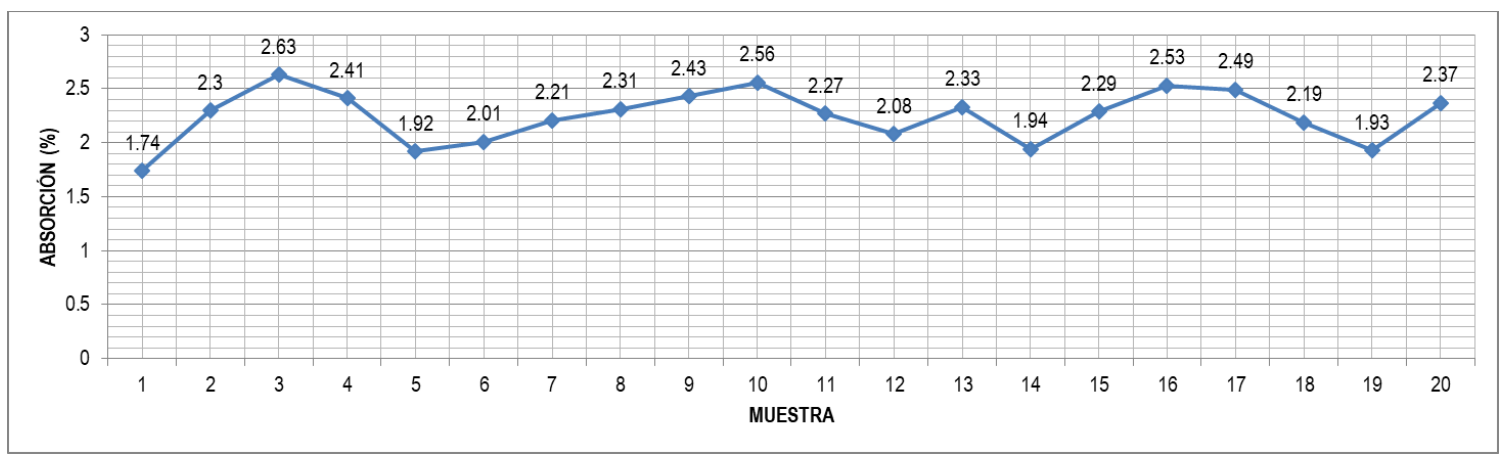

Figura 7.- Resultados de la prueba de humedad y absorción Fuente: Elaboración propia. 


\subsection{Prueba de compresión simple en especímenes}

Los muretes se ensayaron a la edad de 28 días. En la elaboración, curado, transporte, almacenamiento, cabeceado y procedimiento de ensaye de los especímenes se siguió lo estipulado en la Norma Mexicana NMX-C-404-ONNCCE-2012. A continuación, cada uno de los especímenes obtenidos (piezas) se sometió a las pruebas de compresión a 28 días. La Figura 8 ilustra el ensayo de un tabique individual. La Tabla 3 enlista los datos y resultados del ensayo y las Figuras 9 y 10 la gráfica de los mismos.

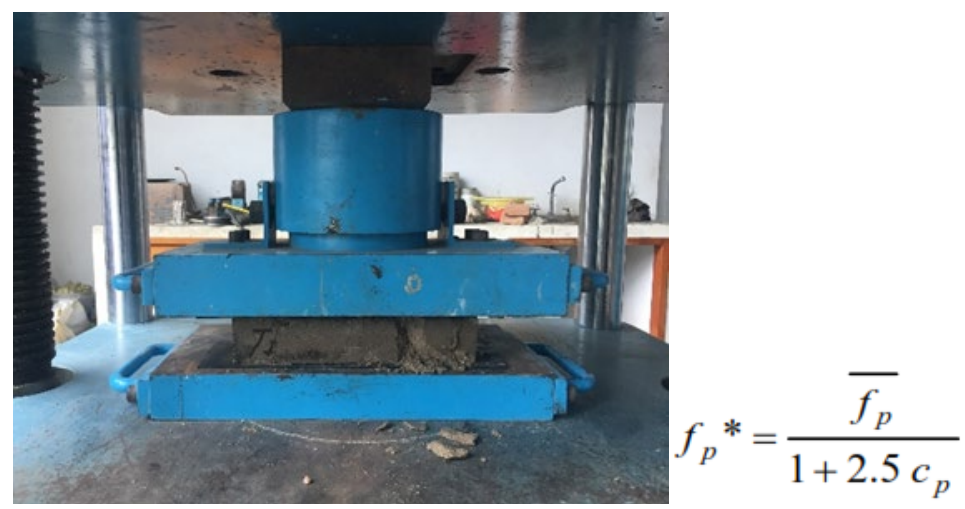

Figura 8.- Prueba de compresión axial en piezas

Fuente: Toma Propia.

Donde:

$\boldsymbol{f}_{\boldsymbol{p}}{ }^{*}$ : Resistencia de diseño a compresión simple en especímenes.

$\overline{\boldsymbol{f}_{\boldsymbol{p}}}$ : Media de la resistencia a compresión de las piezas, referida al área bruta.

$c_{p}$ : Coeficiente de variación de la resistencia a compresión de las piezas.

El valor de $c_{p}$ no se tomará menor que 0.20 para piezas provenientes de plantas mecanizadas que evidencien un sistema de control de calidad como el requerido en la norma NMX-C-404ONNCCE-2012, ni que 0.30 para piezas de fabricación mecanizada, pero que no cuenten con un sistema de control de calidad, ni que 0.35 para piezas de producción artesanal. Los resultados de los ensayos que se obtuvieron para las piezas se resumen en las Tabla 3, en donde se observa que los valores promedio de resistencia de los especímenes individuales (piezas) de tabiques adicionados con plástico tipo 1 PET, es de $193.29 \mathrm{~kg} / \mathrm{cm}^{2}$ con un coeficiente de variación de 0.35 (NTCM, 2017) y teniendo como resistencia de diseño $\left(\mathrm{F}_{\mathrm{p}}{ }^{*}\right) 103.05 \mathrm{~kg} / \mathrm{cm}^{2}$.

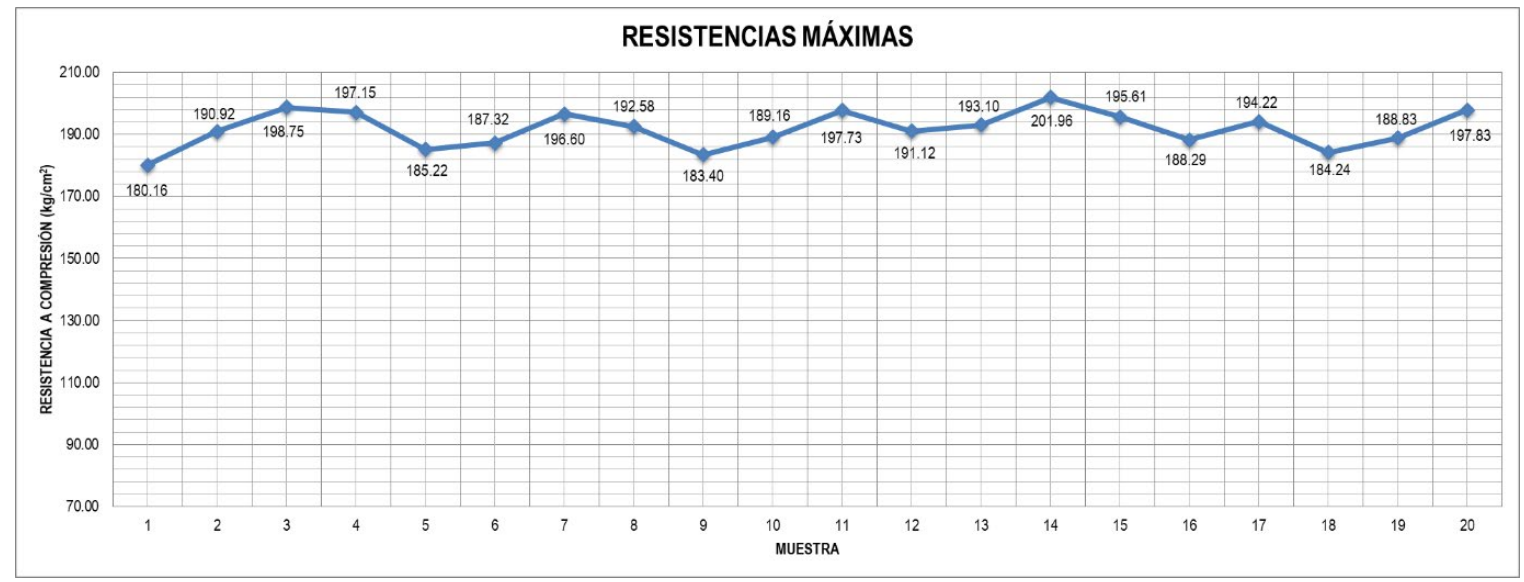

Figura 9.- Resultados de la prueba de compresión axial en piezas

Fuente: Elaboración propia. 


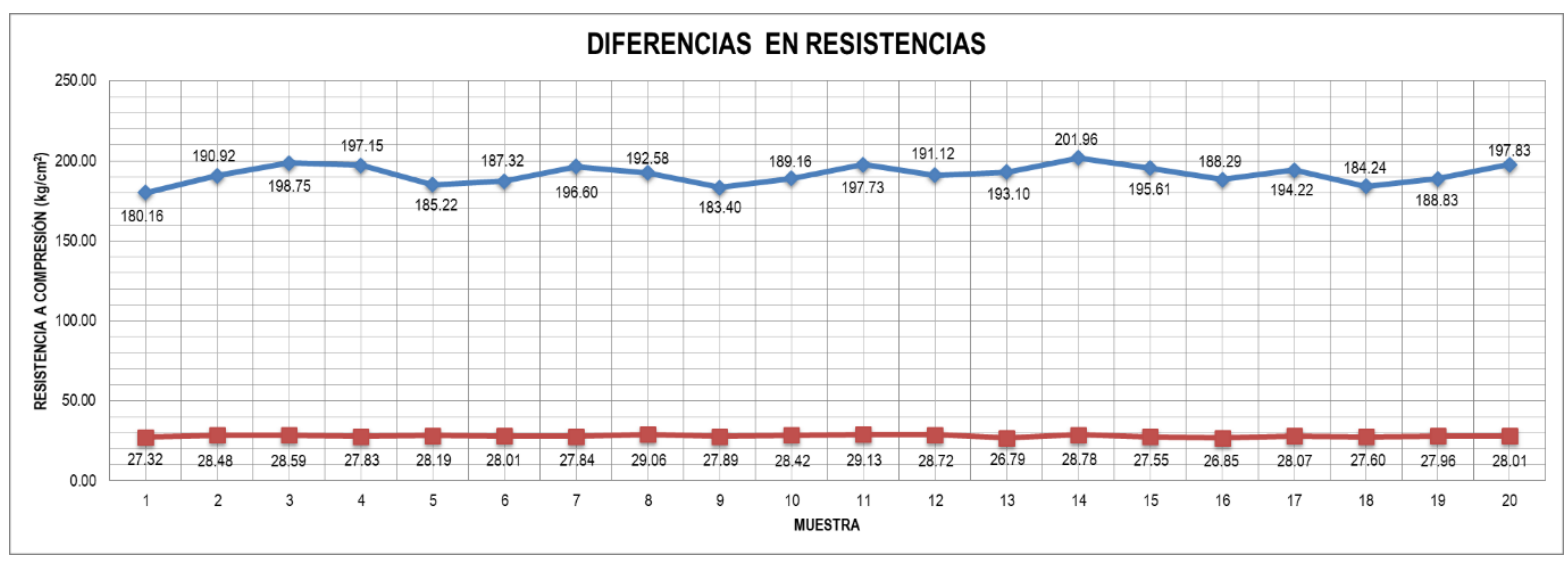

Figura 10.- Diferencias en resistencias, primera grieta y máxima Fuente: Elaboración propia.

Tabla 3.- Resultados de las pruebas de compresión axial en "piezas"

\begin{tabular}{|c|c|c|c|c|c|}
\hline $\begin{array}{l}\text { No. de } \\
\text { muestra }\end{array}$ & $\begin{array}{l}\text { Edad, } \\
\text { Días }\end{array}$ & $\begin{array}{c}\text { Carga a la } \\
\text { primera } \\
\text { grieta, } \mathrm{kg} / \mathrm{cm}^{2}\end{array}$ & $\begin{array}{l}\text { Resistencia a } \\
\text { la primera } \\
\text { grieta, } \mathrm{kg} / \mathrm{cm}^{2}\end{array}$ & $\begin{array}{c}\text { Carga de } \\
\text { ruptura, } \mathrm{kg} / \mathrm{cm}^{2}\end{array}$ & $\begin{array}{l}\text { Resistencia } \\
\text { máxima, } \\
\text { kg/cm² }\end{array}$ \\
\hline 1 & 28 & 7900 & 27.32 & 52100 & 180.16 \\
\hline 2 & 28 & 8100 & 28.48 & 54300 & 190.92 \\
\hline 3 & 28 & 8200 & 28.59 & 57000 & 198.75 \\
\hline 4 & 28 & 7850 & 27.83 & 55600 & 197.15 \\
\hline 5 & 28 & 8050 & 28.19 & 52900 & 185.22 \\
\hline 6 & 28 & 8000 & 28.01 & 53500 & 187.32 \\
\hline 7 & 28 & 7950 & 27.84 & 56150 & 196.60 \\
\hline 8 & 28 & 8300 & 29.06 & 55000 & 192.58 \\
\hline 9 & 28 & 8000 & 27.89 & 52600 & 183.40 \\
\hline 10 & 28 & 8150 & 28.42 & 54250 & 189.16 \\
\hline 11 & 28 & 8250 & 29.13 & 56000 & 197.73 \\
\hline 12 & 28 & 8100 & 28.72 & 53900 & 191.12 \\
\hline 13 & 28 & 7650 & 26.79 & 55150 & 193.10 \\
\hline 14 & 28 & 8150 & 28.78 & 57200 & 201.96 \\
\hline 15 & 28 & 7900 & 27.55 & 56100 & 195.61 \\
\hline 16 & 28 & 7700 & 26.85 & 54000 & 188.29 \\
\hline 17 & 28 & 8050 & 28.07 & 55700 & 194.22 \\
\hline 18 & 28 & 7850 & 27.60 & 52400 & 184.24 \\
\hline 19 & 28 & 7950 & 27.96 & 53700 & 188.83 \\
\hline 20 & 28 & 8000 & 28.01 & 56500 & 197.83 \\
\hline \multirow{2}{*}{\multicolumn{5}{|c|}{$\begin{array}{r}\text { Promedio } \\
\text { Desviación Estándar }\end{array}$}} & 193.29 \\
\hline & & & & & 5.30 \\
\hline \multirow{3}{*}{\multicolumn{5}{|c|}{$\begin{array}{r}\text { Media } \\
\text { Coeficiente de Variación } \\
\text { F }_{\mathrm{p}} *(\text { Resistencia de Diseño }) \\
\end{array}$}} & 193.23 \\
\hline & & & & & 0.35 \\
\hline & & & & & 103.05 \\
\hline
\end{tabular}

Fuente: Resultados obtenidos en el Laboratorio de la Facultad de Ingeniería, UNACH, Chiapas. 


\subsection{Prueba de compresión axial en pilas}

Las pilas se ensayaron a la edad de 28 días. En la elaboración, curado, transporte, almacenamiento, cabeceado y procedimiento de ensaye de los especímenes se siguió lo estipulado en la Norma Mexicana NMX-C-464-ONNCCE-2010. Las pilas están formadas por lo menos con tres piezas sobrepuestas. La relación altura a espesor de la pila estará comprendida entre dos y seis; las pilas se ensayarán a la edad de 28 días.

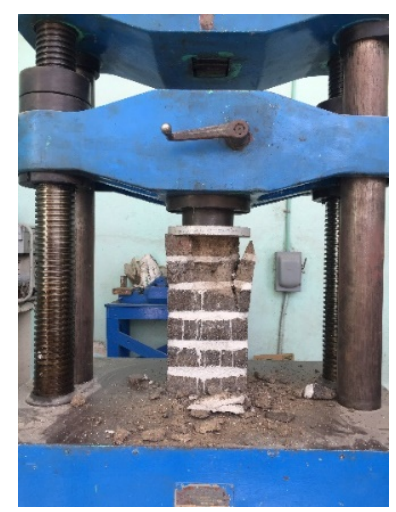

Figura 11.- Prueba de compresión axial en pilas

Fuente: Toma propia.

En la Figura 11 se muestra la pila de tabiques bajo ensayo, en la Figura 12 se muestra el esquema y en la Tabla 4 se muestran las características de las pilas, los resultados de los ensayos a compresión y el tipo de falla observado.

Tabla 4.- Resultados de las pruebas de compresión axial en "pilas"

\begin{tabular}{|c|c|c|c|c|c|c|c|}
\hline $\begin{array}{l}\text { No. de } \\
\text { muestr } \\
\quad \text { a }\end{array}$ & $\begin{array}{c}P_{\max } \\
(\text { carga } \\
\text { máxima), } \\
\quad \mathbf{k g}\end{array}$ & $\begin{array}{c}\text { t } \\
\text { (espesor } \\
\text { de } \\
\text { la pila), } \\
\text { cm }\end{array}$ & $\begin{array}{c}\text { h } \\
\text { (altura de } \\
\text { la pila), } \\
\text { cm }\end{array}$ & $\begin{array}{c}\mathbf{E}=\mathbf{h} / \mathbf{t} \\
\text { (esbeltez) }\end{array}$ & $\begin{array}{c}\text { A } \\
\text { (área de la } \\
\text { sección), } \\
\text { cm }^{2}\end{array}$ & $\begin{array}{c}\mathbf{f}_{\mathrm{m}} \\
\text { (resistencia a } \\
\text { compresión } \\
\text { axial) } \\
\mathbf{k g} / \mathrm{cm}^{2}\end{array}$ & $\begin{array}{c}\text { Tipo de } \\
\text { falla }\end{array}$ \\
\hline 1 & 15000 & 12.00 & 43.00 & 3.58 & 516.00 & 29.07 & Normal \\
\hline 2 & 12100 & 12.20 & 43.00 & 3.52 & 524.60 & 23.07 & Normal \\
\hline 3 & 11500 & 12.10 & 44.00 & 3.64 & 532.40 & 21.60 & Normal \\
\hline 4 & 11000 & 12.00 & 43.50 & 3.63 & 522.00 & 21.07 & Pandeo \\
\hline 5 & 12400 & 12.00 & 43.50 & 3.63 & 522.00 & 23.75 & Normal \\
\hline 6 & 13600 & 12.20 & 43.00 & 3.52 & 524.60 & 25.92 & Normal \\
\hline 7 & 12700 & 12.20 & 43.00 & 3.52 & 524.60 & 24.21 & Normal \\
\hline 8 & 11300 & 12.00 & 44.00 & 3.67 & 528.00 & 21.40 & Pandeo \\
\hline 9 & 14200 & 12.00 & 43.00 & 3.58 & 516.00 & 27.52 & Normal \\
\hline 10 & 12000 & 12.10 & 43.50 & 3.60 & 526.35 & 22.80 & Normal \\
\hline \multirow{5}{*}{\multicolumn{6}{|c|}{$\begin{array}{r}\text { Promedio } \\
\text { Desviación Estándar } \\
\text { Media } \\
\text { Coeficiente de Variación } \\
\text { (Resistencia de diseño) }\end{array}$}} & 24.04 & \\
\hline & & & & & & 2.69 & \\
\hline & & & & & & 23.91 & \\
\hline & & & & & & 0.15 & \\
\hline & & & & & & 17.39 & \\
\hline
\end{tabular}

Fuente: Elaboración propia. 


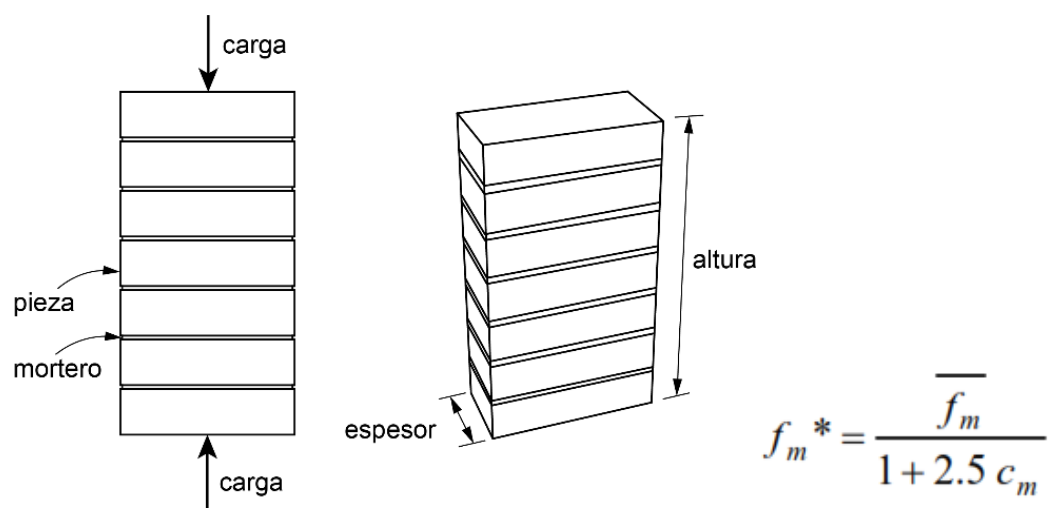

Figura 12.- Representación de la Norma para ensayo a compresión axial en pilas

Fuente: NMX-C-464-ONNCCE-2010

Donde:

$\boldsymbol{f}_{\boldsymbol{m}} \boldsymbol{*}=$ Resistencia de diseño a compresión en pilas.

$\overline{\boldsymbol{f}_{\boldsymbol{m}}}=$ Resistencia media a compresión de las pilas, corregida por su relación altura a espesor y referida al área bruta.

$\boldsymbol{c}_{\boldsymbol{m}}=$ Coeficiente de variación de la resistencia a compresión de las pilas de mampostería, que en ningún caso se tomará inferior a 0.15. (NTCM, 2017)

Como ya se mencionó, los resultados de los ensayos que se obtuvieron para las pilas elaboradas se resumen en las Tabla 6, en donde se observa que los valores promedio de resistencia de las pilas fabricadas con tabiques adicionados con plástico tipo $1 \mathrm{PET}$, es de $24.04 \mathrm{~kg} / \mathrm{cm}^{2}$ con un coeficiente de variación de 0.15 (NTCM, 2017) y una resistencia de diseño $\left(\mathrm{f}_{\mathrm{m}}{ }^{*}\right)$ igual a $17.39 \mathrm{~kg} / \mathrm{cm}^{2}$. La gráfica de la Figura 12 ilustra el comportamiento de compresión axial de las pilas ensayadas.

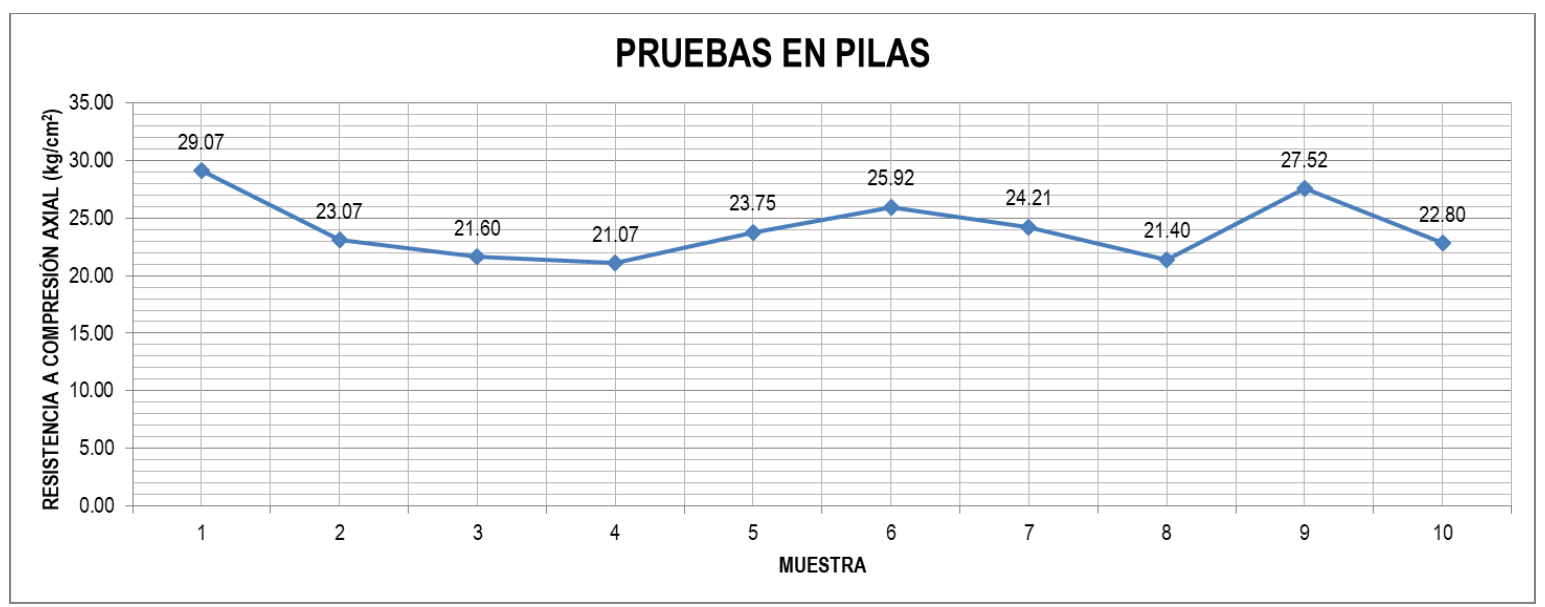

Figura 12.- Resultados de la prueba de compresión axial en pilas Fuente: Elaboración propia.

\subsection{Prueba de compresión diagonal (cortante) en muretes}

Para el caso de muretes se ensayaron a la edad de 28 días. En la elaboración, curado, transporte, almacenamiento, cabeceado y procedimiento de ensaye de los especímenes se siguió lo estipulado en la Norma Mexicana NMX-C-464-ONNCCE-2010. En la Figura 13 se muestra el esquema y la Figura 14 el murete de tabiques bajo ensayo. 


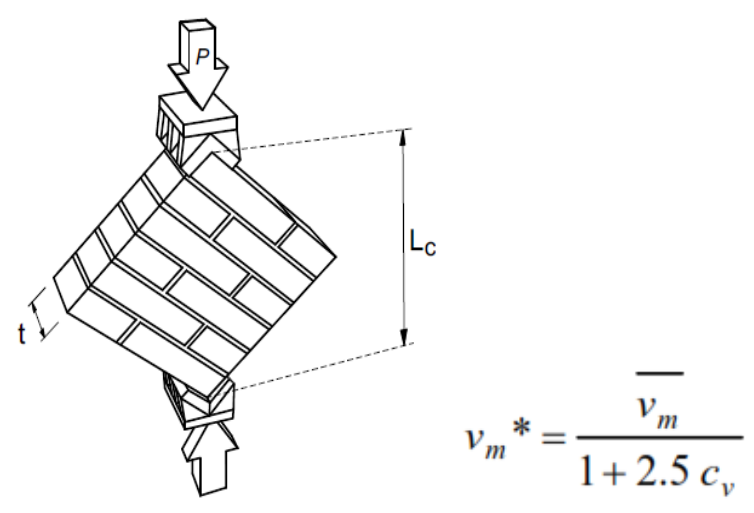

Figura 13.- Representación de la Norma para ensayo a compresión diagonal (cortante) en muretes Fuente: NMX-C-464-ONNCCE-2010

Donde:

$\boldsymbol{v}_{\boldsymbol{m}} \boldsymbol{*}=$ Resistencia de diseño a compresión diagonal en muretes.

$\overline{\boldsymbol{v}_{\boldsymbol{m}}}=$ Resistencia media a compresión diagonal de muretes, sobre área bruta medida a lo largo de la diagonal paralela a la carga.

$\boldsymbol{c}_{\boldsymbol{v}}=$ Coeficiente de variación de la resistencia a compresión diagonal de muretes, que en ningún caso se tomará inferior a 0.20. (NTCM, 2017)

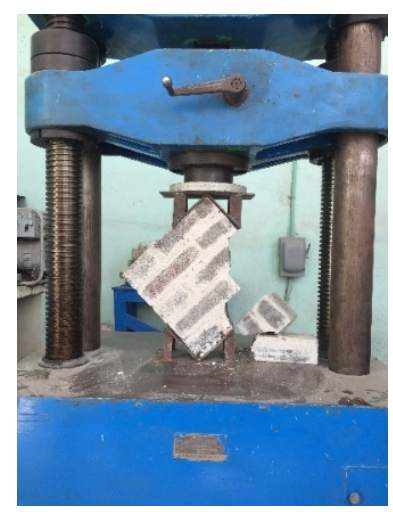

Figura 14.- Prueba de compresión diagonal en muretes

Fuente: Toma propia.

Los resultados de los ensayos que se obtuvieron para los muretes elaborados se resumen en las Tabla 5 y en la Figura 15 la gráfica del mismo, en donde se observa que los valores promedio de resistencia de los muretes fabricados con tabiques adicionados con plástico tipo 1 PET, es de 13.84 $\mathrm{kg} / \mathrm{cm}^{2}$ con un coeficiente de variación de 0.20 (NTCM, 2018) y una resistencia de diseño (Vm*) igual a $9.21 \mathrm{~kg} / \mathrm{cm}^{2}$. 
Tabla 5.- Resultados de las pruebas de compresión diagonal (cortante) en "muretes"

\begin{tabular}{|c|c|c|c|c|c|c|}
\hline $\begin{array}{l}\text { No. de } \\
\text { muestra }\end{array}$ & $\begin{array}{c}P_{\max } \\
\text { (carga } \\
\text { máxima), } \\
\mathbf{k g}\end{array}$ & $\begin{array}{c}\mathbf{t} \\
\text { (espesor del } \\
\text { murete) } \\
\text { cm }\end{array}$ & $\begin{array}{c}\mathbf{L}_{\mathbf{c}} \\
\text { (longitud } \\
\text { de la } \\
\text { diagonal), } \\
\text { cm } \\
\end{array}$ & $\begin{array}{c}\text { A } \\
\text { (área sobre } \\
\text { la diagonal), } \\
\mathbf{c m}^{2}\end{array}$ & $\begin{array}{c}\mathrm{V}_{\mathrm{m}} \\
\text { (resistencia a } \\
\text { compresión } \\
\text { diagonal) } \\
\mathrm{kg} / \mathrm{cm}^{2} \\
\end{array}$ & $\begin{array}{c}\text { Tipo de } \\
\text { falla }\end{array}$ \\
\hline 1 & 7900 & 12.00 & 50.50 & 606.00 & 13.04 & Diagonal \\
\hline 2 & 8250 & 12.20 & 51.00 & 622.20 & 13.26 & Diagonal \\
\hline 3 & 8100 & 12.10 & 51.00 & 617.10 & 13.13 & Diagonal \\
\hline 4 & 9400 & 12.00 & 51.00 & 612.00 & 15.36 & Deslizamiento \\
\hline 5 & 8500 & 12.00 & 50.50 & 606.00 & 14.03 & Diagonal \\
\hline 6 & 7850 & 12.20 & 50.50 & 616.10 & 12.74 & Deslizamiento \\
\hline 7 & 8300 & 12.20 & 51.00 & 622.20 & 13.34 & Diagonal \\
\hline 8 & 8750 & 12.00 & 50.50 & 606.00 & 14.44 & Diagonal \\
\hline 9 & 9100 & 12.00 & 50.50 & 606.00 & 15.02 & Deslizamiento \\
\hline 10 & 8650 & 12.10 & 51.00 & 617.10 & 14.02 & Diagonal \\
\hline \multirow{5}{*}{\multicolumn{5}{|c|}{$\begin{array}{c}\text { Promedio } \\
\text { Desviación Estándar } \\
\text { Media } \\
\text { oeficiente de Variación } \\
\text { (Resistencia de diseño) }\end{array}$}} & 13.84 & \\
\hline & & & & & 0.89 & \\
\hline & & & & & 13.81 & \\
\hline & & & & & 0.20 & \\
\hline & & & & & 9.21 & \\
\hline
\end{tabular}

Fuente: Elaboración propia.

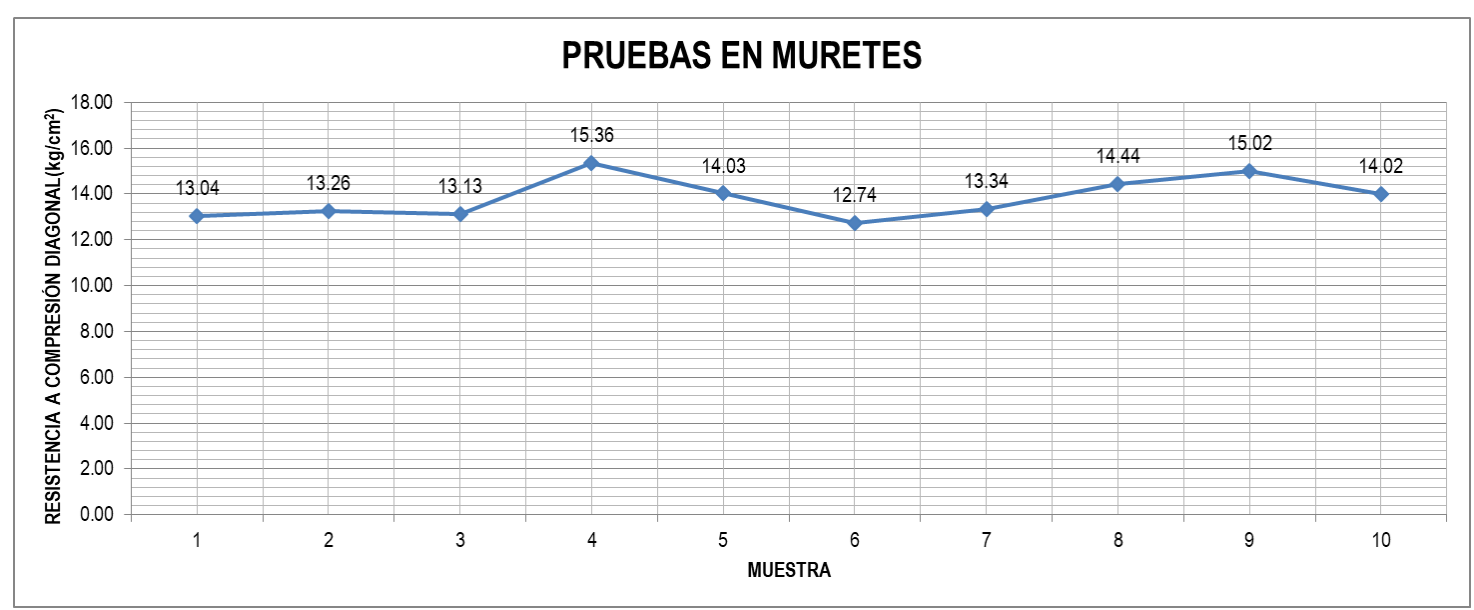

Figura 15.- Pruebas de compresión diagonal en "muretes"

Fuente: Elaboración propia.

\section{CONCLUSIONES}

La utilización de PET, como material aglutinante dentro de la mezcla, demostró ser factible según los resultados obtenidos en esta primera parte del presente estudio, brindando una alternativa de reciclaje que conduce a reducir el impacto ambiental producido por los desechos industriales y urbanos. Es importante señalar que para los fines de la presente investigación se enfocó en conocer las propiedades físicas y mecánicas que brindan estos nuevos materiales. 
La fabricación de PET demanda un consumo energético alto que puede ser recuperado reutilizando dicho material en lugar de desecharlo, esto debido a que su degradación es muy lenta y ocasiona problemas ambientales, motivo por el cual se busca implementarlo como material constructivo no convencional. Cabe resaltar que es escasa la bibliografía relacionada con la mampostería estructural, tampoco estudios realizados a los materiales no convencionales de este trabajo, lo que hace que este proyecto sea una primera aportación en este sentido.

La producción del tabique no convencional en cuestión se hizo de manera artesanal, salvo el hecho de utilizar otros materiales menos comunes como el plástico tipo 1 PET. La selección y cantidad de los materiales que se utilizaron en la elaboración de este tabique se iniciaron de manera inicial empírica e intuitiva, ya que no hay estudios que respalden la fabricación ni las características físicoquímicas de estos materiales empleados, ni proporciones de los materiales, destacando que dentro del proceso no se usa agua, lo que da por resultado un producto que preserva y conserva el vital líquido.

Hoy en día no se encuentra disponible un control de calidad en la producción en ninguna zona estudiada. Las dimensiones del tabique fabricado (6-12-24) son las variables que presentan un coeficiente de variación más bajo, con valores similares y por encima de la zona de estudio, sin embargo no cumplen con las dimensiones mínimas (7-14-28), especificadas por las normas (NMX404-2012-ONNCCE).

Para obtener productos de mejor calidad, que cumplan con la normatividad, se recomienda realizar estudios sobre las características y propiedades físico químicas de los materiales que se utilizan en la fabricación del tabique no convencional, a fin de determinar los tipos de material y el proporcionamiento adecuado de los mismos. Estandarizar y tecnificar el proceso de fabricación para que las características de la producción sean semejantes.

Las condiciones en las que se realizó este estudio fueron modestas, por lo que se recomienda seguir trabajando sobre el tema, promoviendo en el sector público y privado las ventajas de este tipo de investigaciones, dada la importancia e impacto que tiene este material en la construcción masiva de vivienda.

A futuro se pretende mejorar la elaboración de los tabiques, profundizar en los estudios mecánicos y de durabilidad, así como realizar un análisis Emergético y de huella de carbono, para sustentar los costos ambientales derivados del proceso, dado que pueden ser propuestas para líneas de investigación. Los resultados obtenidos en este trabajo son un primer paso del amplio campo que requiere ser estudiado y analizado, como pueden ser entre otros el proceso de fabricación, control de calidad y normatividad del tabique.

Esperando que este esfuerzo sea la base para futuros trabajos que puedan complementar este conocimiento sobre algo tan sencillo en su apariencia pero tan complejo en su estructura y comportamiento, como son las estructuras de mampostería que es utilizada en forma indiscriminada en el Estado de Chiapas, México. 


\section{AGRADECIMIENTOS}

A la Facultad de Ingeniería de la Universidad Autónoma de Chiapas, México, por las facilidades otorgadas al proyecto para su realización y el uso de los diversos laboratorios existentes para llevar a cabo los estudios y ensayos correspondientes, así como también al Laboratorio de Materiales de la Unidad de Servicios Técnicos de la Secretaría de Comunicaciones y Transportes, Delegación Chiapas, México.

\section{REFERENCIAS}

- Alesmar, L., Rendón, N., Korody, M. E. (2008). Diseños de mezcla de tereftalato de polietileno (PET) - cemento. Revista de la Facultad de Ingeniería Universidad Central de Venezuela, 23(1), 76-86.

- Amariz, A. D. M., Jiménez, M. L. C. (2014). Diseño y fabricación de ladrillo reutilizando materiales a base de PET. Inge Cuc, 10(2), 76-80.

- Arroyo, M., Cabrera, O., \& Muñoz, M. (2002). Estudio de las propiedades físicas y mecánicas del tabique rojo recocido, utilizado en la construcción del Municipio de Querétaro. Sociedad Mexicana de Ingeniería Estructural.

- BBC Mundo. (2017). 5 gráficos para entender por qué el plástico es una amenaza para nuestro planeta. Junio 20, 2021, de BBC Mundo News Sitio web: https://www.bbc.com/mundo/noticias-42304901

- Cañizares, F. F., Moreno Cárdenas, R. A. (2011). Agregado alternativo para la elaboración de Bloques y Adoquines a base de Polietilen Tereftalato (Bachelor's thesis, QUITO/EPN/2011).

- Cámara de Diputados H. congreso de Unión. (s.f.). En México, 90 millones de botellas de plástico de refrescos y agua son lanzados a la vía pública, ríos y mares. Recuperado el 30 de Abril de 2019, de http://www5.diputados.gob.mx/index.php/esl/Comunicacion/Boletines/2017/Abril/13/3 469En-Mexico-90-millones-de-botellas-de-plastico-de-refrescos-y-agua-son-lanzados-a-la-viapublica-rios-y-mares

- Criado, M. (20 de 07 de 2017). Los humanos ya han generado 8.300 millones de toneladas de plástico. EL PAIS.

- Flores, L. (2015). Norma Mexicana NMX-C-464-ONNCCE-2010, Ensaye de Muretes y Pilas. Diciembre 21, 2021, de Coordinación Nacional de Protección Civil, México Sitio web: https:/www.smie.org.mx/archivos/eventos/2015/agosto/ponencia-mexico-cambio-parasiempre-desde-1985-norma-mexicana-nmx-c-464-onncce-2010-ensaye-muretes-pilasleonardo-flores.pdf

- Morales, R. O. D. M. (2015). Diseño y Elaboración de un Sistema de Adoquines de Bajo Costo y Material Reciclado para Construcciones en Núcleos Rurales. REVISTA ESAICA, 1(1), 30-38.

- Mexicana, N. NMX-C-404-ONNCCE-2012. - Industria de la construcción - Bloques, tabiques o ladrillo tabicones para uso estructural - especificaciones y métodos de ensayo.

- Mexicana, N. NMX-C-464-ONNCCE-2010. - Industria de la construcción - Mampostería Determinación de la resistencia a compresión diagonal y módulo de cortante de muretes y la resistencia a compresión y módulo de elasticidad de pilas de mampostería de arcilla o de concreto - Métodos de ensayo.

- Morales, C. E., Pérez, B. E. (2018). Determinación de la conductividad térmica y resistencia mecánica de ladrillos y placas conformadas de cemento y polietileno teraftalato (PET) (Bachelor's thesis, Escuela Superior Politécnica de Chimborazo). 
- Piñeros, M. E., Herrera Muriel, R. D. D. J. (2018). Proyecto de factibilidad económica para la fabricación de bloques con agregados de plástico reciclado (PET), aplicados en la construcción de vivienda.

- Quevedo, S. G., Guamán, C. A. (2014). Proyecto de Factibilidad para la Producción de EcoAdoquines Peatonales Mediante la Reutilización de Desechos Plásticos (Pet) (Bachelor's thesis).

- Salazar, J. (01 de 2019). Chiapas no recicla; el plástico llega a ríos. EL DIARIO DE CHIAPAS.

- Subdirección de Legalidad. (2017). Normas Técnicas Complementarias para Diseño y Construcción de Estructuras de Mampostería. Junio 21, 2021, de Gobierno de la Ciudad de México, Secretaría de la Contraloría General Sitio web: http://www3.contraloriadf.gob.mx/prontuario/index.php/normativas/Template/ver_mas/65838/ $39 / 1 / 1$

- Zavala, G. (2015). Diseño y desarrollo experimental de materiales de construcción utilizando plástico reciclado. Informe final de investigación. Escuela especializada en ingeniería itcafepade dirección de investigación y proyección social.

- Zea, J, Alonso, F. A., Mandujano, J. E. (2017). "Diseño y elaboración de adoquines no convencionales destinados al tránsito ligero mediante la reutilización de desechos plásticos". Congreso Mesoamericano de Investigación UNACH, 1472-1476.

- Zúñiga, A. (2015). Evaluación del tereftalato de polietileno (PET) como agregado en la elaboración de mortero para ladrillos y concreto. 7,8 . 